\title{
Prepare for the Worst - Using Facility Location to Alleviate Product Recall Risks
}

\author{
Liufang Yao (Corresponding author) \\ Faculty of Business, University of Prince Edward Island \\ McDougall Hall, Room 323, 550 University Avenue, Charlottetown, PE, C1A 4P3, Canada \\ E-mail: 1yao@upei.ca
}

Andrew G. Carrothers

Faculty of Business, University of Prince Edward Island

McDougall Hall, Room 323, 550 University Avenue, Charlottetown, PE, C1A 4P3, Canada

Tel: 1-902-330-8011Ｅ-mail: acarrothers@upie.ca

Received: October 20, 2020 Accepted: November 16, 2020 Published: December 2, 2020

doi:10.5296/ber.v10i4.18024 URL: https://doi.org/10.5296/ber.v10i4.18024

\begin{abstract}
This paper is motivated by major food product recall events in recent years, especially how the timely and effective response using post-recall management can make a difference. We consider the rare but very influential major product recalls as disruptions to the supply chain and incorporate locating reprocessing centers for the returned products to mitigate expected operational costs. We adopt the closed loop network design framework and assume the location decisions for reprocessing center take place after the product recall events. Our scenario-based analysis shows the approach is effective in both absolute and relative measures.
\end{abstract}

Keywords: Risk mitigation, Product recall risks, Facility location model, Forward and reverse flow logistics, Lagrangian relaxation

\section{Introduction}

In September 2012, the rejection of ground beef imports by the U.S. custom and the later outbreak of the E.coli disease forced XL Inc. (XL) to start a series of beef recalls, which turned out to be the largest meat recall event in Canada history. The recall of 1,800 products impacted over 33 retail chains across Canada. Over 4,000 tons of meat and meat products 
were sent back to plants for disposal. This unprecedented amount of recalled products overwhelmed the capacities of any existing disposal methods of XL, the largest domestically owned meat processor in Canada at the time, resulting in 600 tons of frozen beef being sent directly to landfill. The failure to adequately process recalled products raised the public concern over XL's capability to maintain food safety. This incident eventually led to a transfer of XL's ownership for the Calgary plant. Charlebois et al. (2015) discussed this event and its impacts in detail.

In comparison, when facing the 2008 Listeria outbreak, Maple Leaf Food Inc. (MLF) recalled all potentially affected products promptly and dealt with the disposal of 1,300 tons of beef and beef products. MLF's actions bought time to discover the contamination source and recover the brand. Nevertheless, there was a substantial direct cost of $\$ 19$ million related to recall activities (e.g., collection and destruction, shutdown and sanitation of facility, media, and customer response call center). In total, MLF suffered approximately $\$ 200$ million loss in this incident.

Food safety is generally referred to as the prevention of illness resulting from the consumption of contaminated food as discussed by Akkerman et al. (2010). This topic has attracted more attention recently because of the growing rigorous government standards as well as the large social and financial impacts of major food safety failure. Effective control of food safety along the supply chain is important but very complicated because food is vulnerable to contamination and food supply chains are sophisticated. For example, Desmarchelier et al. (2007) provide a summary of food safety management in the red meat industry of Australia. Risk mitigation strategies are applied in the entire food supply chain.

However, food borne disease is a critical inherent risk factor in food manufacturing and distribution, due to the indigenous existence of microbial contaminations in raw food materials, natural growth of pathogens, inevitable mistakes in manual operations, contamination, and other factors. In response to food safety incidents, business and society adopt food recalls to correct the situation and mitigate monetary and social costs. Manufacturing companies manage food recalls by collecting products from their distribution channels and adopting best methods to recondition and dispose recalled products.

Proper preparation could help companies manage food recalls more efficiently and effectively, especially in strategic planning. For example, firms could use location-allocation decisions for both manufacturing plants and reprocessing facilities for recalled products. Linking to optimization studies, Akkerman et al. (2010) provide a review of improving food supply chain management with network planning models. Food quality, food safety and sustainability are considered as key objectives. Three levels of network planning models are considered, namely strategic network design (e.g., facility location-allocations), tactical network planning (e.g., production and distribution) and operational transportation planning (e.g., routing). They suggest that strategic network design is critical in food safety control, impacting how long food products travel and how widely the products spread geographically, both of which determine the size of potential product recalls.

This paper addresses the supply chain safety control issue by designing the supply chain 
network to incorporate the negative effects of product recalls. Although our model is motivated by food recall, it can be applied to any supply chain with significant impacts of recall events. Extending from the closed-loop network design concept, this work focuses on managing the reverse flow (recalled products) in a cost-efficient manner. However, rather than maintaining a closed-loop supply chain on a daily basis as in most studies (e.g., repair and post-sale service systems), we study the efficient way of managing random and rare major product recalls. The features of rareness and randomness of major products recalls lead us to disruption management studies in which researchers focus on how to consistently satisfy customer demands given that some suppliers may fail. Our focus is different in that we consider how to quickly build a reprocessing network to dispose recalled products.

We study the location-allocation problem with random occurrence of product recalls and treat the recall incidences as disruptions to the supply chain. In our setting, the company first makes decisions to locate manufacturing plants and allocate demands. After the product recall occurs, we make decisions to locate the reprocessing center(s) from internal (self-owned recall facility) or external (third-party business) sources and allocate recalled products for reprocessing or local disposal. Three features distinguish our problem from other location-allocation problems. Firstly, facility location and allocation decisions occur in two stages. Secondly, the second stage location-allocation happens under uncertainty. Thirdly, reverse logistic flows exist in the second stage.

We design a two-stage stochastic mixed integer programming model, in which we locate the manufacturing plants in the first stage and the reprocessing/disposal facilities in the second stage. We adopt a scenario-based approach to describe the uncertainty of major recall events that may happen in manufacturing plants as well as of availability of reprocessing facilities. Given the complexity induced by our nested facility location problem, we devise an algorithm based on Lagrangian relaxation to solve the uncapacitated case.

This paper will be organized as follows. Section 2 provides a brief review of literature. Section 3 introduces the mathematical model, an analysis based on facility capacities, and a Branch-and-Bound algorithm incorporating Lagrangian relaxation for the uncapacitated case. Section 5 presents the computational results and managerial insights from experiments. Section 6 summarizes contributions and discusses further research directions.

\section{Literature Review}

There are two research streams closely related to our research, i.e., reliable supply chain network design, and location-allocation with bidirectional logistical flows.

There is a well-developed literature on modeling supply chain disruption management. Snyder et al. (2006) provide a review of optimization models in supply chain network planning with disruption management. They categorize by network status, underlying mathematical models and risk measures. They show various models extending the classical P-median and Uncapacitated Facility Location Problem (UFLP), Capacitated Facility Location Problem (CFLP) models with reliability features (i.e., consistent satisfaction of demands when some facilities fail in random disruptions). Qi et al. (2010) consider a 
fortification model with disruptions. They manage locations, allocations, and inventory. Both suppliers and retailers can experience random disruptions. Qi (2013) examines different sourcing and replenishment decisions with two suppliers. Dada et al. (2007) develop a newsvendor procurement model selecting from multiple unreliable suppliers. Their results suggest that newsvendor, customers, and retailers perceive different service level changes when disruption occurs. Compared to reliability concerns, cost is the most determinant factor. This body of work focuses on satisfying customer demand - there is no focus on reverse flow.

On the other hand, in closed-loop supply chain models, the emphasis is to minimize the long-run average cost of forward and reverse flows, which does not model the random occurrence of product recalls. Uncertainty in closed-loop supply chain network design is not always considered. Some studies use a fixed return rate based on historical data (e.g., Lee \& Dong, 2008; Lu \& Bostel, 2007; Min \& Ko, 2008; Salema et al., 2006). Savaskan et al. (2004) describe product return rate as a function of investment used to promote product return.

Hitherto, uncertain factors in closed-loop supply chain include return rate, demand for re-manufactured products (secondary market), quality of returned products, and variable costs for collection, processing and transportation. Pishvaee et al. (2011) describe uncertain demands, returns and transportation costs given by a robust network design model. Salema et al. (2007) address uncertain demand and return with scenario dependent uniform random numbers to minimize the total cost of the reverse logistics network. Ramezani et al. (2013) consider uncertainty in demand and return ratio as well as various variable costs in their multi-objective forward/reverse network design. Listeş (2007) uses scenario-dependent parameters to describe uncertain demand and returned product quantity for their supply and product-return networks. Few researchers assume the scenario of randomly failing manufacturing plants which results in major recall events.

The literature has taken different approaches to deciding the quantity to dispose during returned products collection and reprocessing. Early literature tends to not consider disposal cost. For instance, Savaskan et al. (2004) do not take disposal as a cost factor when comparing different re-manufacturing channels. With increasing focus on environment-friendly and efficient supply chains, disposal costs are reflected in later studies. For example, Min and Ko (2008) alter the repair facility capacities to accommodate returned quantities at each time period so no disposal will occur.

Decisions regarding disposal cost can be categorized in three types: fixed ratios, market driven, and cost driven. Fixed disposal ratios are deduced from historical data and adopted to simplify the model (e.g., Lee \& Dong, 2008; Lu \& Bostel, 2007; Pishvaee et al., 2011). Cost driven decisions aim to minimize total cost of collection, reprocessing and disposal. Salema et al. (2007) minimize total supply chain costs by using fraction of customer demand used for disposal or recovery. Ramezani et al. (2013) use disposed quantity to maximize the total profit within the capacity of opened disposal centers. Market driven decisions select the best efforts to satisfy demands of secondary market. Pishvaee et al. (2011) model the disposal quantity as decision variable so that secondary market can be satisfied in the most efficient 
way. Listeş (2007) takes the perspective that returned products can be disposed in two decisions: before collection and before reprocessing, both of which aim to maximize the total profit while satisfying market demands.

In summary, the existing literature treats reverse flow on day-to-day basis. This modeling approach does not serve major product recalls well. Our challenge is to design an optimal network that can accommodate product returns in the context of major product recalls.

\section{Facility Location to Mitigate Recall Risks}

\subsection{Model Development}

In this section, we use a two-stage stochastic programming approach described by Ruszczynski and Shapiro (2003) to model the problem. In the first stage, we make facility location and transportation decisions. In the second stage, under each disruption scenario, we make recall decisions including recall facility locations (e.g., centers for reconditioning, reprocessing, and rendering) and recall allocation decisions (e.g., use local disposal or recall center). The objective is to minimize the sum of facility location costs, transportation costs and recall costs.

Define $\mathcal{J}$ as the set of candidate locations for manufacturing facilities and use $i$ as the index. Define $\mathcal{K}$ as the set of candidate locations for recall centers and use $k$ as the index. Note that recall centers could reuse the manufacturing facilities or use third party processing facilities. Thus, we could have $\mathcal{J} \subset \mathcal{K}$ or $\mathcal{J} \cap \mathcal{K}=\emptyset$. We use $F F$ and $R F$ to indicate forward product flow and reverse product flow (initiated by recall events) respectively. Clearly, facilities built for manufacturing and recall processing have different fixed costs, defined as $f_{i}^{F F}$ and $f_{k}^{R F}$. Facility capacity is denoted by $M_{i}^{F F}$ or $M_{k}^{R F}$.

Define $\mathcal{J}$ as the set of retailers and use $j$ as the index. Each retailer has a demand $D_{j}$, the cost of shipping one unit of product demand from facility $i$ to retailer $j$ is $c_{i j}^{F F}$, while the reverse flow costs $c_{j k}^{R F}$ per unit. For recalled products, two recall modes are available, i.e., local disposal and central processing. Local disposal incurs a retailer location related cost $c_{j}^{L D}$, and central processing incurs a recall center related cost $c_{i}^{C P}$.

To describe the uncertainty of facility disruption, we use scenario set $\mathcal{S}$ and index $s$. We used $p_{s}$ to denote the probability of scenario $s$. Manufacturing facilities failing in scenario $s$ is denoted by set $\mathcal{J}_{s}$. Accordingly, recall centers available in scenario $s$ is denoted by set $\mathcal{K}_{s}$. The choice of $\mathcal{K}_{s}$ can be decided extraneously. For instance, recalled products could be prohibited from returning to their original manufacturing facility due to safety concerns, or be sent to third party facilities due to economic considerations.

Decision variables used in this model are facility location variables ( $\mathbf{X}$ for manufacturing facilities and $\mathbf{Z}$ for recall facilities), transportation variables $(\mathbf{Y})$, and recall assignment variables ( $\mathbf{V}$ for local disposal and $\mathbf{W}$ for central processing): 


$$
\begin{gathered}
X_{i}= \begin{cases}1 & \text { manufacturing facility at location } i \text { is open } \\
0 & \text { otherwise. }\end{cases} \\
Z_{k s}= \begin{cases}1 & \text { recall facility is open at location } k \text { under scenario } s \\
0 & \text { otherwise. }\end{cases}
\end{gathered}
$$

$Y_{i j}$ : quantity transported from facility $i$ to retailer $j$

$V_{j s}$ : quantity for local disposal under scenario $s$ at retailer $j$

$W_{j k s}$ : quantity from retailer $j$ to plant $k$ for central processing under scenario $s$

With these notations, the two-stage stochastic program for the Facility Location with Recall Problem $(\mathcal{F} \mathcal{L} \mathcal{R P})$ is formulated as follows:

$$
\begin{aligned}
& (\mathcal{F} \mathcal{L} \mathcal{R P}) \min \\
& \sum_{i \sim 7} f_{i}^{F F} X_{i}+\sum_{i \sim n} \sum_{i \sim 7} c_{i j}^{F F} Y_{i j}
\end{aligned}
$$

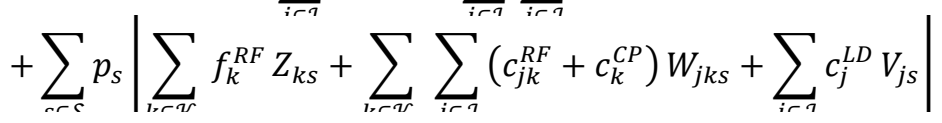

$$
\begin{aligned}
& \text { s.t. } \quad \sum_{i=r} Y_{i j}=D_{j} \quad \forall j \in \mathcal{J} \\
& \sum_{i \sim \boldsymbol{I}} Y_{i j} \leq M_{i}^{F F} X_{i} \quad \forall i \in \mathcal{J} \\
& \sum_{i\ulcorner r} W_{j k s} \leq M_{k}^{R F} Z_{k s} \quad \forall s \in S, k \in \mathcal{K}_{s} \\
& \sum_{l, \sim \mathcal{T}} W_{j k s}+V_{j s}=\sum_{i \sim r} Y_{i j} \quad \forall j \in \mathcal{J}, s \in \mathcal{S} \\
& Y_{i j} \geq 0 \quad \forall i \in \mathcal{J}, j \in \mathcal{J} \\
& W_{j k s}, V_{j s} \geq 0 \quad \forall j \in J, s \in \mathcal{S}, k \in \mathcal{K}_{s} \\
& X_{i} \in\{0,1\} \quad \forall i \in \mathcal{J} \\
& Z_{k s} \in\{0,1\} \quad \forall s \in \mathcal{S}, k \in \mathcal{K}_{s}
\end{aligned}
$$

The objective (1) is the expected total cost of facility location-allocation decisions from both stages. In the first stage, the manufacturing facility location and demand distribution are determined, while in second stage, recall center location and recall product distribution are determined. Constraint (2) ensures demand of each retailer is satisfied. Constraint (3) and (4) guarantees forward and recalled products are processed in an open facility within the capacities. Constraint (5) requires recalled products either be sent back to recall center for reprocessing or disposed locally. Other constraints are for non-negativity and for binary variables.

It is noteworthy that the proposed formulation is very general and can include different recall situations. Firstly, the model can cover cases of capacitated and uncapacitated facilities or any hybrid types by changing the capacity parameter $M_{i}^{F F}$ and $M_{k}^{R F}$ to be infinite or finite numbers. Secondly, the definition of set $\mathcal{K}_{s}$ provides a lot of flexibility in modeling. For instance, facilities in $\mathcal{K}_{s}$ can be the exact locations where the food safety incidents happen, 
suggesting that recalled products must return to their original manufacturing plants; a type of corrective action in this situation is to fix problems such as mislabeling. We can also require facilities in $\mathcal{K}_{s}$ be locations other than the original manufacturing facilities to model the situation in which the original facilities are unsuitable for processing (e.g., safety overhaul). Moreover, $\mathcal{K}_{s}$ could be third party facility locations to model the outsourcing of processing recalled products. Thirdly, for further extension, recall facility location decisions $Z_{k s}$ 's could be moved to the first stage. The difference between this extension and $(\mathcal{F} \mathcal{L} \mathcal{R P})$ will show the difference between proactive recall facility location decisions and reactive facility location decisions.

In addition, the model could indicate different traceability capability in forward flow. Currently, we assume full traceability in forward flow, i.e., each retailer could distinguish the products from failed plants from others. We can also model incomplete traceability such that retailer can't identify the source of the recalled products, by altering constraint (5) to $\sum_{k \in \mathcal{K}_{s}} W_{j k s}+V_{j s}=\sum_{i \in \mathcal{J}} Y_{i j}$ for all $j \in \mathcal{J}$ and $s \in \mathcal{S}$.

\subsection{Analysis}

If the demand of a retailer is satisfied by more than one facility, it is called "demand splitting", otherwise, it is called "no demand splitting". Note that $(\mathcal{F} \mathcal{L} \mathcal{R} \mathcal{P})$ contains two types of demands, corresponding to the two stages (i.e., customer demand and recall demand). To be concise, we use "plants" to denote manufacturing centers located for forward flows, and "facilities" to denote processing centers located for reverse flows. We have the following result in the optimal demand splitting schemes of $(\mathcal{F} \mathcal{L} \mathcal{R P})$ :

Theorem 1. In the optimal solutions of $(\mathcal{F} \mathcal{L} \mathcal{R P})$, demand splitting schemes of forward and reverse flows depend on the capacity constraints of both plants and facilities. There are four schemes:

(a) If plants and facilities are both uncapacitated, then there exists no demand splitting for both forward and reverse flows;

(b) If plants are capacitated but facilities are uncapacitated, then there exists demand splitting for forward flow and no demand splitting for reverse flow;

(c) If plants are uncapacitated but facilities are capacitated, then there could exist demand splitting for both flows;

(d) If plants and facilities are both capacitated, then there could exist demand splitting for both flows.

Proof. Consider an arbitrary retailer $u \in \mathcal{J}$. Assume that except the allocation decisions for $u$, all other decisions are fixed to their optimal values. We use superscript $*$ to represent the optimal values. These decisions include:

$X_{i}^{*}(i \in \mathcal{J})$ locating plants for forward flow,

$Z_{k s}^{*}\left(k \in \mathcal{K}_{s}, s \in \mathcal{S}\right)$ locating facilities for reverse flow, 
$Y_{i j}^{*}(i \in I, j \in \mathcal{J} \backslash\{u\})$ allocating demands of retailers other than $u$ in forward flow,

$W_{j k s}^{*}, V_{j s}^{*}\left(j \in \mathcal{J} \backslash\{u\}, k \in \mathcal{K}_{s}, s \in \mathcal{S}\right)$ allocating recall demands of retailers other than $u$ for central reprocessing and local disposal respectively in reverse flow.

In the following, we discuss the demand splitting schemes in two steps. We isolate demand splitting decisions in the reverse flow in the first step, and apply the results in the second step to integrate both forward and revers flows. Note we use $*$ to denote optimal solutions for $(\mathcal{F} \mathcal{L} \mathcal{R} \mathcal{P})$ in this proof, while later in algorithm design we use $*$ to denote best solutions found in Lagrangian relaxation.

Step 1. Demand allocations in forward flows, i.e., $Y_{i u}^{*}(i \in \mathcal{J})$, are known and optimal.

We use $\mathcal{K}_{s}^{*}$ to denote the set of facilities to open for reverse flow in scenario $s \in \mathcal{S}$ in the optimal solution. Let $\beta_{k s}$ be the proportion of demand for retailer $u$ allocated to facility $k \in \mathcal{K}_{s}^{*}$ in scenario $s, \beta_{s}^{\prime}$ be the proportion of demand allocated to local disposal in scenario $s$. We have $\sum_{k \in K_{s}^{*}} \beta_{k s}+\beta_{s}^{\prime}=1$ in scenario $s$.

Let $D_{u s}^{*}$ be the optimal recall demand from $u$ in scenario $s$. Note that when forward flow is traceable, we have $D_{u s}^{*}=\sum_{i \in \mathcal{J}_{s}} Y_{i u}^{*}$; when forward flow is untraceable, we have $D_{u s}^{*}=$ $\sum_{i \in \mathcal{J}} Y_{i u}^{*}$ if $Y_{i u}^{*}>0$ for some $i \in \mathcal{J}_{s}$, and $D_{u s}^{*}=0$ otherwise.

Using $\Omega$ to denote the total cost of known location and allocation decisions, $(\mathcal{F} \mathcal{L R \mathcal { P }})$ can be simplified as follows:

$$
\begin{gathered}
\min \sum_{s \in \mathcal{S}} p_{s}\left[\sum_{k \in \mathcal{K}_{s}^{*}}\left(c_{u k}^{R F}+c_{k}^{C P}\right) \beta_{k s} D_{u s}+c_{u}^{L D} \beta_{s}^{\prime} D_{u s}\right] \\
\text { s.t. } \sum_{k \in \mathcal{K}_{s}^{*}} \beta_{k s}+\beta_{s}^{\prime}=1, \forall s \in \mathcal{S} \\
\qquad \beta_{k s} D_{u s}+\sum_{j \in \mathcal{J} \backslash\{u\}} W_{j k s}^{*} \leq M_{k}^{R F}, \forall k \in \mathcal{K}_{s}^{*}, s \in \mathcal{S} \\
\beta_{k s}, \beta_{s}^{\prime} \geq 0, \forall k \in \mathcal{K}_{s}^{*}, s \in \mathcal{S} .
\end{gathered}
$$

Note that local disposal at retailer $u$ always has unlimited capacity. This setting can be altered by adding constraint $\beta_{s}^{\prime} D_{u s} \leq M_{u}^{R F}$ in the analysis, where $M_{u}^{R F}$ is the capacity for local disposal of $u$.

If the facilities are uncapacitated, the optimal solution is either 0 or 1 because the objective function is a linear function of $\beta_{k s}$ and $\beta_{s}^{\prime}$ defined on interval $[0,1]$. In other words, there 


\section{Macrothink}

is no demand splitting for any scenario $s \in \mathcal{S}$. Denote

$$
\gamma_{s}^{*}=\min \left\{c_{u}^{L D}, \min _{k \in \mathcal{K}_{s}^{*}}\left\{c_{u k}^{R F}+c_{k}^{C P}\right\}\right\}
$$

and optimal recall cost of $u$ as $\psi_{s}^{*}\left(D_{u s}^{*}\right)=\gamma_{s}^{*} D_{u s}^{*}$, then the optimal objective value is $\Omega+\sum_{s \in \mathcal{S}} p_{s} \psi_{s}^{*}\left(D_{u s}^{*}\right)$.

If the facilities are capacitated, in order to minimize the objective function, we sort the coefficients $\left(c_{u k}^{R F}+c_{k}^{C P}\right)\left(k \in \mathcal{K}_{s}^{*}\right)$ and $c_{u}^{L D}$ in increasing order, denoted as $c_{1}, c_{2}, \cdots, c_{n_{s}+1}$ where $n_{s}=\left|\mathcal{K}_{s}^{*}\right|$. Then the optimal solution is to assign demand according to this order given the capacity constraints. We redefine reverse flow cost $\psi_{s}^{*}\left(D_{u s}^{*}\right)$ as follows:

$$
\psi_{s}^{*}\left(D_{u s}^{*}\right)= \begin{cases}c_{1} D_{u s}^{*} & D_{u s}^{*} \leq M_{1}^{R F} \\ c_{k+1}\left(D_{u s}^{*}-\sum_{i=1}^{k} M_{i}^{R F}\right)+\sum_{i=1}^{k} c_{i} M_{i}^{R F} & \sum_{i=1}^{k} M_{i}^{R F} \leq D_{u s}^{*} \leq \sum_{i=1}^{k+1} M_{i}^{R F}, \forall k=1, \cdots, n_{s}\end{cases}
$$

where $c_{1} \leq c_{2} \leq \cdots \leq c_{n_{s}} \leq c_{n_{s}+1}$. Note that if the unit cost of local disposal is ranked before that of facility $j$, then calculations for facility $j$ and its followers are unnecessary because local disposal is uncapacitated.

We have the same form of optimal objective value $\Omega+\sum_{s \in \mathcal{S}} p_{s} \psi_{s}^{*}\left(D_{u s}^{*}\right)$. Note that reverse flow cost $\psi_{s}^{*}\left(D_{u s}^{*}\right)$ is a continuous and nondecreasing piece-wise linear function of $D_{u s}^{*}$ when facility capacities are limited. Clearly $\psi_{s}^{*}(\cdot)$ is a convex function.

Step 2. In the optimal solution, denote $\mathcal{J}^{*}$ as the set of plants open in forward flow, $\mathcal{J}_{S}^{*}$ as the set of plants that are open and get disrupted in scenario $s \in \mathcal{S}$. Let $\alpha_{i}$ be the proportion of demand allocated to plant $i \in \mathcal{J}^{*}$ for forward flows, $\psi_{s}^{*}\left(D_{u s}^{*}\right)$ be the optimal cost for reverse flow as defined in Step 1, where $D_{u s}^{*}=\sum_{i \in J_{s}^{*}} \alpha_{i} D_{u}$ (or in the case with untraceable demand, if $Y_{i u}^{*}>0$ for some $i \in \mathcal{J}_{s}^{*}$, then $\left.D_{u s}^{*}=D_{u}\right)$. Let $\Psi$ be the cost of all known optimal decisions. $(\mathcal{F} \mathcal{L} \mathcal{R} \mathcal{P})$ can be simplified as follows:

$\min$

$$
\Psi+\sum_{i \in \mathcal{J}^{*}} c_{i u}^{F F} \alpha_{i} D_{u}+\sum_{s \in \mathcal{S}} p_{s} \psi_{s}^{*} \sum_{i \in \mathcal{J}_{s}^{*}}\left(\alpha_{i} D_{u}\right)
$$

s.t.

$$
\begin{gathered}
\sum_{i \in \mathcal{J}^{*}} \alpha_{i}=1 \\
\alpha_{i} D_{u}+\sum_{j \in \mathcal{J} \backslash\{u\}} Y_{i j}^{*} \leq M_{i}^{F F}, \forall i \in \mathcal{J}^{*} \\
\alpha_{i} \geq 0, \forall i \in \mathcal{J}^{*}
\end{gathered}
$$


Proof. If facilities are uncapacitated, then $\psi_{s}^{*}\left(D_{u s}^{*}\right)=\gamma_{s}^{*} D_{u s}^{*}=\gamma_{s}^{*} \sum_{i \in J_{s}^{*}} \alpha_{i} D_{u}$. Clearly, the objective function is linear in $\alpha_{i}$ 's. It holds that when plants are uncapacitated, the plant incurring the lowest cost will be chosen, thus the optimal solution does not have demand splitting for neither forward nor reverse flows, which completes the proof for Theorem 1 Scheme (a). On the other hand, with capacitated plants the optimal demand splitting scheme for reverse flow remains to be no demand splitting; while capacity limits of plants in forward flow require demand splitting to satisfy all demands at the lowest cost, which completes the proof for Theorem 1 Scheme (b).

In the case of capacitated facilities and uncapacitated plants, apparently demand splitting in reverse flow exists because of facility capacity limits, would the demand splitting in reverse flow lead to demand splitting in forward flow even when there is no restriction on plant capacity?

Suppose, on the contrary, there is no demand splitting in the optimal decision. Consider the retailer $u$ has demand $D_{u}$, served by two plants $\mathcal{J}^{*}=\left\{i_{1}, i_{2}\right\}$ and two facilities $\mathcal{K}_{s}^{*}=$ $\left\{k_{1}, k_{2}\right\}$ that are open for all scenarios. Plants fail independently. Let us compare the following two cases.

In the first case, $D_{u}$ is completely served by plant $i_{1} \in \mathcal{J}^{*}$. Denote the probability of failure of $i_{1}$ as $q_{i_{1}}=\sum_{s: i_{1} \in J_{s}^{*}} p_{s}$, called the failure rate of $i_{1}$. The allocation cost $T C_{1}$ for $i_{1}$ can be represented as $T C_{1}=c_{i_{1} u}^{F F} D_{u}+q_{i_{1}} \psi\left(D_{u}\right)$. Note we use $\psi(\cdot)$ instead of $\psi^{*}(\cdot)$ because it is not optimal solution. In the second case, $D_{u}$ is satisfied by two plants $i_{1}$ and $i_{2}$. Let $\alpha_{i_{1}}$ and $\alpha_{i_{2}}$ denote the proportions of demand $D_{u}$ satisfied by $i_{1}$ and $i_{2}$ respectively, where $\alpha_{i_{1}}+\alpha_{i_{2}}=1$. Let the failure rates of plants $i_{1}$ and $i_{2}$ be $q_{i_{1}}$ and $q_{i_{2}}$ respectively. Then the total allocation cost $T C_{2}$ would be $C_{2}=$

$\alpha_{i_{1}} c_{i_{1} u}^{F F} D_{u}+\alpha_{i_{2}} c_{i_{2} u}^{F F} D_{u}+q_{i_{1}}\left(1-q_{i_{2}}\right) \psi\left(\alpha_{i_{1}} D_{u}\right)+\left(1-q_{i_{1}}\right) q_{i_{2}} \psi\left(\alpha_{i_{2}} D_{u}\right)+q_{i_{1}} q_{i_{2}} \psi\left(D_{u}\right)$.

Comparing $T C_{1}$ and $T C_{2}$ :

$$
\begin{aligned}
T C_{1}-T C_{2}= & \left(1-\alpha_{i_{1}}\right) c_{i_{1} u}^{F F} D_{u}-\alpha_{i_{2}} c_{i_{2} u}^{F F} D_{u}-q_{i_{1}}\left(1-q_{i_{2}}\right) \psi\left(\alpha_{i_{1}} D_{u}\right) \\
& -\left(1-q_{i_{1}}\right) q_{i_{2}} \psi\left(\alpha_{i_{2}} D_{u}\right)+q_{i_{1}}\left(1-q_{i_{2}}\right) \psi\left(D_{u}\right) \\
= & \alpha_{i_{2}}\left(c_{i_{1} u}^{F F}-c_{i_{2} u}^{F F}\right) D_{u}+\left(q_{i_{1}}-q_{i_{2}}\right) \psi\left(\alpha_{i_{2}} D_{u}\right) \\
& +q_{i_{1}}\left(1-q_{i_{2}}\right)\left[\psi\left(D_{u}\right)-\psi\left(\alpha_{i_{1}} D_{u}\right)-\psi\left(\alpha_{i_{2}} D_{u}\right)\right] .
\end{aligned}
$$

Because $\psi(\cdot)$ is nondecreasing and convex, we have $\psi\left(D_{u}\right)-\psi\left(\alpha_{i_{1}} D_{u}\right)-\psi\left(\alpha_{i_{2}} D_{u}\right) \geq 0$. If $c_{i_{1} u}^{F F}-c_{i_{2} u}^{F F}=q_{i_{1}}-q_{i_{2}}=0$, then $T C_{1}-T C_{2} \geq 0$. Otherwise, by setting external parameters, we can always construct a problem where in the optimal solution, demand splitting is better than no demand splitting.

In more general case with multiple (more than two) plants and facilities available to serve the 
retailer, we can also construct a problem which has an optimal solution with demand splitting, which completes the proof for Scheme (c). In addition, a numerical example is given in Example 2. Finally, when plants and facilities are both capacitated, from the same analysis, the claim in Scheme (d) holds.

The most interesting result in Theorem 1 is the existence of demand splitting in forward flow when the (reverse flow) facilities are capacitated, as shown in Scheme (c). We use a numerical example to illustrate Theorem 1.

Example 2. Suppose we have all the information of the optimal solution except demand splitting scheme for retailer $u$. With demand $D_{u}^{*}=10$, retailer $u$ has two plants $\mathcal{J}^{*}=\{1,2\}$ available in forward flow, and two facilities $\mathcal{K}_{s}^{*}=\{3,4\}$ for any scenario $s \in \mathcal{S}$ available in reverse flow. We assume the manufacturing facilities fail independently with probability $q_{1}=0.1$ and $q_{2}=0.9$ respectively. We have four scenarios (with probability of $p_{1}=$ $0.81, p_{2}=p_{3}=0.09, p_{4}=0.01$ ) for all possible outcomes.

Transportation unit costs in forward flow are $c_{1 u}^{F F}=1, c_{2 u}^{F F}=30$, unit cost of transportation and central processing for reverse flow are $c_{u 3}^{R F}+c_{u 3}^{C P}=2, c_{u 4}^{R F}+c_{u 4}^{C P}=60$, local disposal unit cost is $c_{u}^{L D}=100$. Suppose plants and local disposal location have unlimited capacity, and capacities of central processing centers are $M_{3}^{R F}=5, M_{4}^{R F}=60$.

We use TC to represent the expected total allocation cost of $u$. Note facility 3 and 4 are capable of processing any amount of returned product from $u$, thus the local disposal at $u$ need not to be considered (more expensive), which leaves TC to be solely dependent on the demand splitting factor $\alpha_{1}$, i.e., the proportion of $D_{u}$ satisfied by plant 1 in forward flow. With no demand splitting, customer $u$ is solely served by plant 1 with TC $=289$.

$$
\begin{aligned}
\alpha_{1} & =1 \\
T C & =\sum_{i=1}^{2} c_{i u}^{F F} \alpha_{i} D_{u}++\sum_{s \in \mathcal{S}} p_{s} \psi_{s}\left(\sum_{i \in J_{s}^{*}} \alpha_{i} D_{u}\right) \\
& =1 \cdot 10+0.81 \cdot(5 \cdot 2+5 \cdot 60)+0.09 \cdot(5 \cdot 2+5 \cdot 60) \\
& =289
\end{aligned}
$$

Similarly, when $u$ is solely supplied by plant 2 we have TC $=355.8$. However, if the demand is shared by the two plants evenly, we have $T C=191.9$. The results show demand splitting scheme with $\alpha_{1}=0.5$ gives lower cost than no demand splitting $\left(\alpha_{1}=1\right.$ or $\left.\alpha_{1}=0\right)$. Moreover, solving the problem with MAPLE shows that $\alpha_{1}=0.5$ is the optimal solution, which complies with our statement in Theorem 1 Scheme (c).

\section{Lagrangian Relaxation}

To find the optimal solution for model $(\mathcal{F} \mathcal{L} \mathcal{R P})$, we could use readily available commercial solvers such as CPLEX. However, preliminary computational studies show that this problem is hard to solve even for medium size instances. This motivates us to develop a more efficient method based on Lagrangian relaxation.

Major difficulties for solving $(\mathcal{F} \mathcal{L} \mathcal{R} \mathcal{P})$ come from two aspects: 1) the demand balance constraint (2) impedes us from developing an analytical algorithm similar to that of Snyder 


\section{Macrothink}

and Daskin (2005); 2) constraint (5) increases the complexity further by connecting the forward flow and the reverse flow. For clarity, we introduce the binary parameter $\delta_{i s}$ equals one to denote the case plant $i$ has triggered recall event in scenario $s$, and zero for otherwise. We could relax constraints (2) and (5) of $(\mathcal{F} \mathcal{L} \mathcal{R P})$ by introducing Lagrangian multipliers $\lambda=\left(\lambda_{j}\right)$ and $\boldsymbol{\mu}=\left(\mu_{j s}\right)$ to obtain the following Lagrangian relaxation problem:

$$
\left(\mathcal{F} \mathcal{L} \mathcal{R P}-\mathcal{L R}_{\lambda, \mu}\right)
$$

$\min \mathcal{L}(\boldsymbol{\lambda}, \boldsymbol{\mu})$

$$
\begin{gathered}
=\sum_{i \in \mathcal{J}} f_{i}^{F F} X_{i}+\sum_{i \in \mathcal{J}} \sum_{j \in \mathcal{J}} c_{i j}^{F F} Y_{i j}+\sum_{j \in \mathcal{J}} \lambda_{j}\left(D_{j}-\sum_{i \in \mathcal{J}} Y_{i j}\right) \\
+\sum_{s \in \mathcal{S}} p_{s}\left[\sum_{k \in \mathcal{K}_{s}} f_{k}^{R F} Z_{k s}+\sum_{k \in \mathcal{K}_{s}} \sum_{j \in \mathcal{J}}\left(c_{j k}^{R F}+c_{k}^{C P}\right) W_{j k s}+\sum_{j \in \mathcal{J}} c_{j}^{L D} V_{j s}\right] \\
+\sum_{s \in \mathcal{S}} \sum_{j \in \mathcal{J}} \mu_{j s}\left(\sum_{i \in \mathcal{J}_{S}} Y_{i j}-\sum_{k \in \mathcal{K}_{s}} W_{j k s}-V_{j s}\right) \\
=\sum_{i \in \mathcal{J}} f_{i}^{F F} X_{i}+\sum_{i \in \mathcal{J}} \sum_{j \in \mathcal{J}}\left(c_{i j}^{F F}-\lambda_{j}+\sum_{s \in \mathcal{S}} \mu_{j s} \delta_{i s}\right) Y_{i j}+\sum_{j \in \mathcal{J}} \lambda_{j} D_{j} \\
+\sum_{s \in \mathcal{S}} \sum_{k \in \mathcal{K}_{s}} p_{s} f_{k}^{R F} Z_{k s}+\sum_{s \in \mathcal{S}} \sum_{k \in \mathcal{K}_{s}} \sum_{j \in \mathcal{J}}\left[p_{s}\left(c_{j k}^{R F}+c_{k}^{C P}\right)-\mu_{j s}\right] W_{j k s} \\
+\sum_{s \in \mathcal{S}} \sum_{j \in \mathcal{J}}\left(p_{s} c_{j}^{L D}-\mu_{j s}\right) V_{j s}
\end{gathered}
$$

s.t.

(3), (4), (8), (9)

$$
\begin{gathered}
0 \leq Y_{i j} \leq D_{j}, \forall i \in \mathcal{J}, j \in \mathcal{J} \\
0 \leq W_{j k s} \leq D_{j}, \forall j \in \mathcal{J}, s \in \mathcal{S}, k \in \mathcal{K}_{s} \\
0 \leq V_{j s} \leq D_{j}, \forall j \in \mathcal{J}, s \in \mathcal{S}, k \in \mathcal{K}_{s} .
\end{gathered}
$$

Constraint (11) is modified from (6) to confine that the product quantity delivered to retailer $j$ is no more than the retailer's demand. Similarly, constraints (12) and (13) are modified from constraint (7) to confine local disposal and central processing quantities.

\subsection{Lower Bound}

Relaxing constraint (5) breaks up the connection between forward and reverse flows, 


\section{Macrothink}

therefore we can separate $\left(\mathcal{F} \mathcal{L} \mathcal{R} \mathcal{P}-\mathcal{L} \mathcal{R}_{\lambda, \mu}\right)$ into two optimization problems, i.e., $\left(\mathcal{F} \mathcal{L} \mathcal{R} \mathcal{P}-\mathcal{L} \mathcal{R}_{\lambda, \mu}-\mathcal{F}\right)$ for forward flow and $\left(\mathcal{F} \mathcal{L} \mathcal{R} \mathcal{P}-\mathcal{L} \mathcal{R}_{\lambda, \mu}-\mathcal{R}\right)$ for reverse flow, which can be solved separately. The optimization problem for the forward flow is defined as:

$$
\left(\mathcal{F} \mathcal{L} \mathcal{R} \mathcal{P}-\mathcal{L} \mathcal{R}_{\lambda, \mu}-\mathcal{F}\right)
$$

$\min$

$$
\sum_{i \in \mathcal{J}} f_{i}^{F F} X_{i}+\sum_{i \in \mathcal{J}} \sum_{j \in \mathcal{J}}\left(c_{i j}^{F F}-\lambda_{j}+\sum_{s \in \mathcal{S}} \mu_{j s} \delta_{i s}\right) Y_{i j}+\sum_{j \in \mathcal{J}} \lambda_{j} D_{j}
$$

s.t.

(3), (8), (11).

Note that $\left(\mathcal{F} \mathcal{L} \mathcal{R} \mathcal{P}-\mathcal{L} \mathcal{R}_{\lambda, \mu}-\mathcal{F}\right)$ can be solved by an efficient algorithm. We will open a plant at site $i$ if and only if this decision decrease objective value. Objective value changes due to opening a plant at $i$ (denoted by $\phi_{i}$ ) can be determined by solving the following optimization problem:

$$
\begin{gathered}
\phi_{i}=\min f_{i}^{F F}+\sum_{j \in \mathcal{J}}\left(c_{i j}^{F F}-\lambda_{j}+\sum_{s \in \mathcal{S}} \mu_{j s} \delta_{i s}\right) Y_{i j} \\
\text { s.t. } \sum_{j \in \mathcal{J}} Y_{i j} \leq M_{i}^{F F} \text {, and (11). }
\end{gathered}
$$

The calculation of $\phi_{i}$ depends on whether plant $i$ has capacity limits.

In uncapacitated case $\left(M_{i}^{F F}=\infty\right)$, we have:

$$
Y_{i j}^{*}= \begin{cases}D_{j}, & c_{i j}^{F F}-\lambda_{j}+\sum_{s \in \mathcal{S}} \mu_{j s} \delta_{i s}<0 \\ 0, & \text { otherwise. }\end{cases}
$$

In capacitated case $\left(M_{i}^{F F}<\infty\right)$, the problem is a continuous knapsack problem and we can find the optimal solution greedily as the method presented in Daskin (2011). For plant $i$, sort customer $j$ in increasing order of $\left(c_{i j}^{F F}-\lambda_{j}+\sum_{s \in \mathcal{S}} \mu_{j s} \delta_{i s}\right)$. Let $j^{\prime}$ be the new ranking, and $\mathcal{J}^{-}$be the set of customers with negative coefficients. Then:

$$
Y_{i j^{\prime}}^{*}= \begin{cases}\min \left\{D_{j^{\prime}}, M_{i}^{F F}-\sum_{m=1}^{j^{\prime}-1} D_{m}\right\}, & j^{\prime} \in \mathcal{J}^{-} \\ 0, & \text { otherwise. }\end{cases}
$$

Next, the optimization problem for the reverse flow is defined as:

$$
\begin{gathered}
\left(\mathcal{F} \mathcal{L} \mathcal{R} \mathcal{P}-\mathcal{L} \mathcal{R}_{\lambda, \mu}-\mathcal{R}\right) \\
\min \quad \sum_{s \in \mathcal{S}} \sum_{k \in \mathcal{K}_{s}} p_{s} f_{k}^{R F} Z_{k s}+\sum_{s \in \mathcal{S}} \sum_{k \in \mathcal{K}_{s}} \sum_{j \in \mathcal{J}} \hat{c}_{j k s} W_{j k s}+\sum_{j \in \mathcal{J}} \sum_{s \in \mathcal{S}}\left(p_{s} c_{j}^{L D}-\mu_{j s}\right) V_{j s}
\end{gathered}
$$


s.t.

(4), (9), (12), (13),

where $\quad \hat{c}_{j k s}=p_{s}\left(c_{j k}^{R F}+c_{k}^{C P}\right)-\mu_{j s} \quad$ for $\quad$ all $j \in \mathcal{J}, s \in \mathcal{S}, k \in \mathcal{K}_{s}$. Clearly, $(\mathcal{F} \mathcal{L} \mathcal{R} \mathcal{P}-$ $\left.\mathcal{L R}_{\lambda, \mu}-\mathcal{R}\right)$ is separable for $s \in \mathcal{S}, k \in \mathcal{K}_{s}$. Also notice that local disposal decisions $\mathbf{V}=\left(V_{j s}\right)$ can be decided independently of $\mathbf{Z}=\left(Z_{k s}\right)$ and $\mathbf{W}=\left(W_{j k s}\right)$ as follows:

$$
V_{j s}^{*}= \begin{cases}D_{j}, & p_{s} c_{j}^{L D}-\mu_{j s}<0 \\ 0, & \text { otherwise }\end{cases}
$$

Given a scenario $s$, if we locate a facility at candidate site $k \in \mathcal{K}_{s}$, changes of objective value (denoted by $\psi_{k s}$ ) are determined by the following optimization problem:

$$
\begin{array}{cc}
\psi_{k s}=\min & p_{s} f_{k}^{R F}+\sum_{j \in J} \hat{c}_{j k s} W_{j k s} \\
\text { s.t. } & \sum_{j \in \mathcal{J}} W_{j k s} \leq M_{k}^{R F} .
\end{array}
$$

To solve $\left(\mathcal{F} \mathcal{L} \mathcal{R P}-\mathcal{L} \mathcal{R}_{\lambda, \mu}-\mathcal{R}\right)$, we open a facility $k$ under scenario $s$ if and only if $\psi_{k s}<0$. The solution of (16) depends on whether plants have capacity limits.

In uncapacitated case $\left(M_{k}^{R F}=\infty\right)$, we have:

$$
W_{j k s}^{*}= \begin{cases}D_{j}, & \hat{c}_{j k s}<0 \\ 0, & \text { otherwise }\end{cases}
$$

In capacitated case $\left(M_{k}^{R F}<\infty\right)$, sort customer $j$ in increasing order of $\hat{c}_{j k s}$. Let $j^{\prime}$ be the new ranking, and $\mathcal{J}^{-}$be the set of customers with negative $\hat{c}_{j k s}$. Then:

$$
W_{j k s}^{*}= \begin{cases}\min \left\{D_{j^{\prime}}, M_{k}^{R F}-\sum_{m=1}^{j^{\prime}-1} D_{m}\right\}, & j^{\prime} \in \mathcal{J}^{-} \\ 0, & \text { otherwise. }\end{cases}
$$

\subsection{Upper Bound}

We solve $(\mathcal{F} \mathcal{L} \mathcal{R P})$ using location decisions obtained from $\left(\mathcal{F} \mathcal{L} \mathcal{R} \mathcal{P}-\mathcal{L} \mathcal{R}_{\lambda, \mu}\right)$ for an upper bound. With location decisions, $(\mathcal{F} \mathcal{L} \mathcal{R} \mathcal{P})$ is reduced to a linear programming problem with allocation decisions only. However, in cases of capacitated plants, initial location decisions may be infeasible to obtain an upper bound problem due to insufficient plants' capacity. To restore feasibility, we design a greedy heuristic procedure by assigning unfixed closed plants to open in increasing order of contribution $\phi(i)$ (shown in the following algorithm).। 


\section{Mll Macrothink}

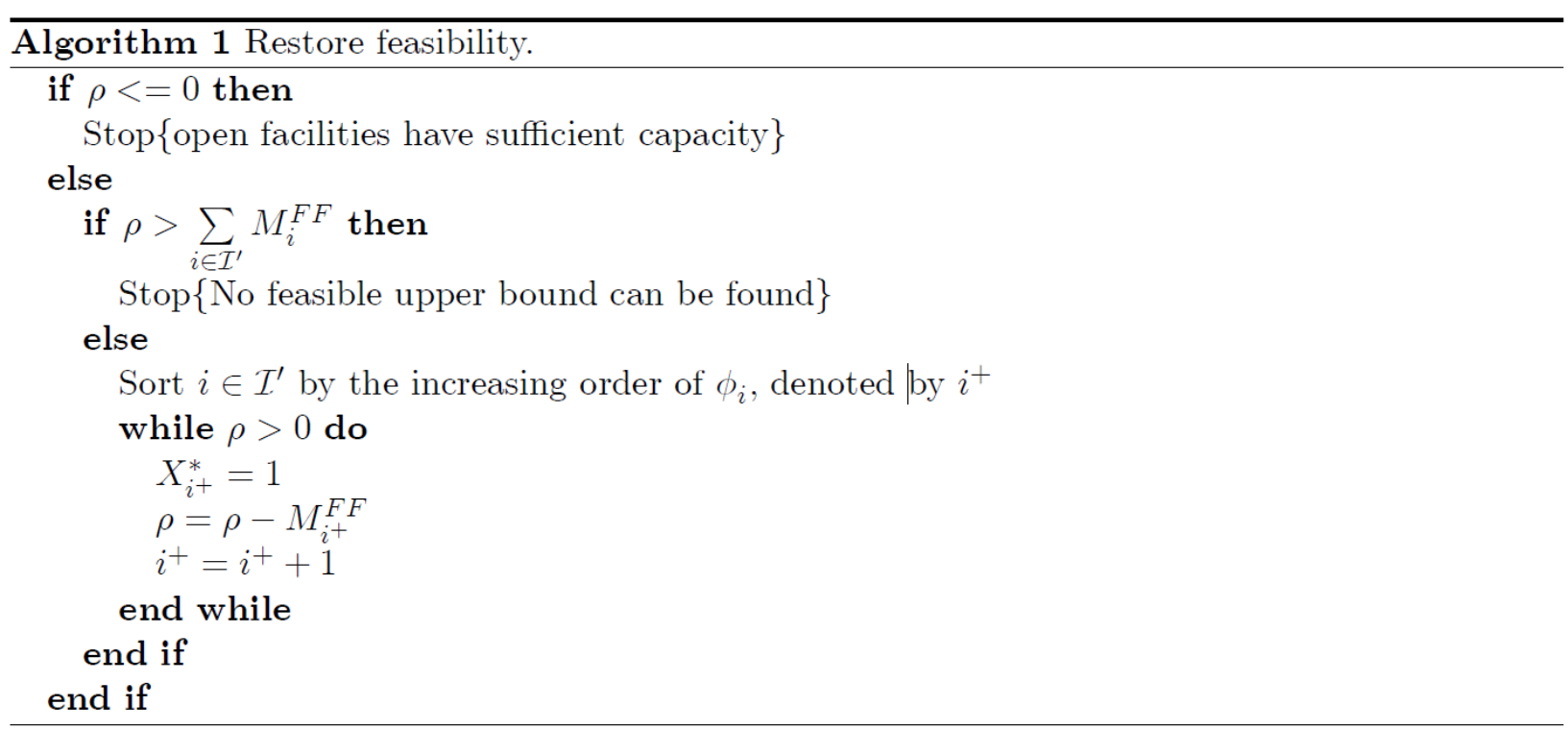

Let $\mathcal{J}^{\prime}$ be the set of plants that are closed in the optimal solution of relaxed problem $\left(\mathcal{F} \mathcal{L R} \mathcal{P}-\mathcal{L R}_{\lambda, \mu}\right)$ but not fixed to closure (e.g. in later mentioned branching process of branch and bound), and denote demands not fulfilled by total capacities of open plants with

$$
\rho=\sum_{j \in \mathcal{J}} D_{j}-\sum_{i \in \mathcal{J}^{\prime}} M_{i}^{F F}
$$

Note that we do not need to adjust location solutions of reverse flows, because local disposal has unlimited capacity and any returned products beyond capacities of central reprocessing can be handled with local disposal method.

Let $\hat{\mathcal{J}}$ be the set of all open plants and set $\widehat{\mathcal{K}}_{s}$ denotes all open facilities in scenario $s$ in the optimal solution of relaxed problem $\left(\mathcal{F} \mathcal{L} \mathcal{R} \mathcal{P}-\mathcal{L} \mathcal{R}_{\lambda, \mu}\right)$. The problem to obtain upper bound is formulated as follows:

$\min$

$$
\sum_{i \in \mathcal{J}} \sum_{j \in \mathcal{J}} c_{i j}^{F F} Y_{i j}+\sum_{s \in \mathcal{S}} \sum_{j \in \mathcal{J}} p_{s}\left[\sum_{k \in \mathcal{K}_{s}}\left(c_{j k}^{R F}+c_{k}^{C P}\right) W_{j k s}+c_{j}^{L D} V_{j s}\right]
$$

s.t.

$$
\text { (2), (5), (6), (7) }
$$

$$
\begin{gathered}
\sum_{j \in J} Y_{i j} \leq M_{i}^{F F} X_{i}^{*}= \begin{cases}M_{i}^{F F}, & i \in \hat{\mathcal{J}} \\
0, & i \in I-\hat{\mathcal{J}}\end{cases} \\
\sum_{j \in J} W_{j k s} \leq M_{k}^{R F} Z_{k s}^{*}= \begin{cases}M_{k}^{R F}, & k \in \widehat{\mathcal{K}}_{s}, s \in \mathcal{S} \\
0, & k \in \mathcal{K}-\widehat{\mathcal{K}}_{s}, s \in \mathcal{S}\end{cases}
\end{gathered}
$$

With constraint (5) maintaining flow balance at different scenarios, above problem cannot be categorized as a transportation problem. With Theorem 1 for Schemes (a) and (b) where facilities' capacities are uncapacitated, costs incurred in stage two can migrate to stage one 
and constraint (5) is omitted.

In Schemes (a) and (b) with uncapacitated facilities, returned products of affected customer are fully processed in one recall mode (either local disposal or central processing). Optimal recall mode is selected based on comparative economic attractiveness. Let set $J_{s+}=\cup_{s \in \mathcal{S}} J_{S}$ denote plants that fail in at least one scenario. Let the unit cost for reverse flow at each customer $j$ in scenario $s$ in the upper bound solution be $\tilde{c}_{j s}$, where $\tilde{c}_{j s}=\min \left\{\min _{k \in \widehat{\mathcal{K}}_{s}}\left\{c_{j k}^{R F}+c_{k}^{C P}\right\}, c_{j}^{L D}\right\}$.

Allocation decisions in reverse flows can be calculated directly:

$$
\begin{gathered}
W_{j k s}^{*}=\left\{\begin{array}{ll}
\sum_{i \in \hat{\jmath} \cap \jmath_{s+}} Y_{i j}, & c_{j k}^{R F}+c_{k}^{C P}=\tilde{c}_{j s} \\
0, & \text { otherwise }
\end{array}, V_{j s}^{*}=0 ;\right. \\
\text { OR } \\
V_{j s}^{*}=\left\{\begin{array}{ll}
\sum_{i \in \hat{\jmath} \cap \jmath_{s+}} Y_{i j}, & c_{j}^{L D}=\tilde{c}_{j s} \\
0, & \text { otherwise }
\end{array}, W_{j k s}^{*}=0\right.
\end{gathered}
$$

Then the transportation problem can be transformed into:

$$
\begin{array}{r}
\min _{(2),(6),(8)} \sum_{i \in \mathcal{J}} \sum_{j \in \mathcal{J}} c_{i j}^{F F} Y_{i j}+\sum_{s \in \mathcal{S}} \sum_{j \in J} p_{s} \tilde{c}_{j s} \sum_{i \in \mathcal{J}}\left(\delta_{i s} Y_{i j}\right) \\
=\sum_{i \in \hat{\mathcal{J}}} \sum_{j \in J}\left(c_{i j}^{F F}+\sum_{s \in \mathcal{S}} p_{s} \tilde{c}_{j s} \delta_{i s}\right) Y_{i j} .
\end{array}
$$

In Scheme (a) where manufacturing plants are uncapacitated, there is no demand splitting in both forward and reverse flows according to Theorem 1 . Thus allocation decisions are either demand $D_{j}$ or 0 . We can calculate the optimal upper bound given solutions of the Lagrangian relaxation problem $\left(\mathcal{F} \mathcal{L} \mathcal{R} \mathcal{P}-\mathcal{L} \mathcal{R}_{\lambda, \mu}\right)$ with the following algorithm. 


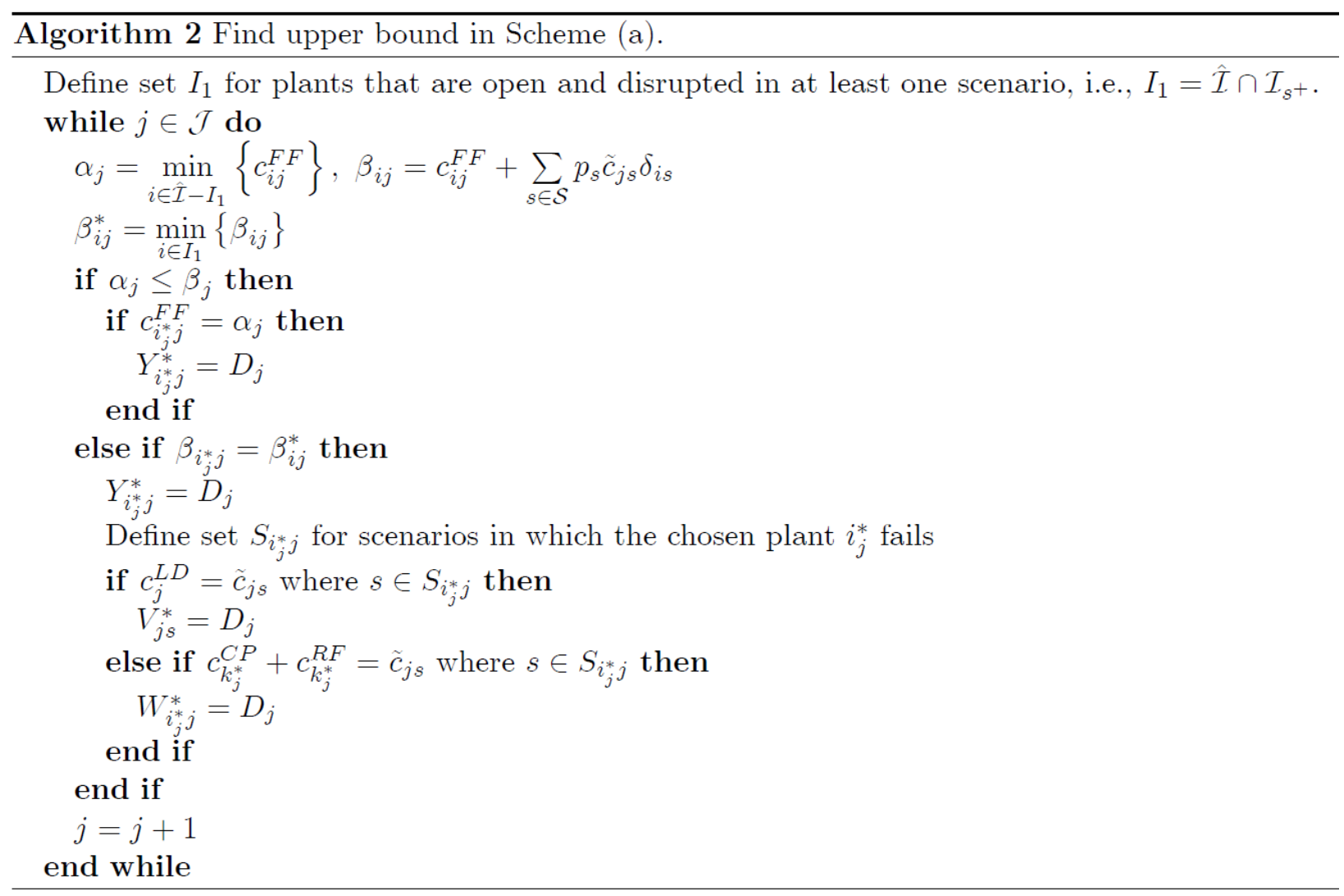

In Scheme (b), problem (20) fit the classic form of transportation problem and can be solved by network simplex method. We refer to the work of Chvátal (1983) that provides a good guidance for network simplex method.

We close plants and facilities that serve no customers in the found upper bound solution.

In cases Scheme (c) and (d), we use Cplex LP solver.

\subsection{Lagrangian Multipliers}

Each vector pair of $(\boldsymbol{\lambda}, \boldsymbol{\mu})$ forms a lower bound $\mathcal{L}(\boldsymbol{\lambda}, \boldsymbol{\mu})$ to the optimal solution of $(\mathcal{F} \mathcal{L} \mathcal{R P})$. To obtain the optimal solution, we need to solve

$$
\max _{\lambda, \mu} \mathcal{L}(\boldsymbol{\lambda}, \boldsymbol{\mu})
$$

We use the subgradient method to update the Lagrangian multipliers as in Fisher (1981). In the $n$th iteration of Lagrangian relaxation algorithm, denote the lower bound with $\mathcal{L}^{n}$, the best upper bound found so far with $B U B$, and the Lagrangian multipliers for the next iteration with $\lambda^{n+1}, \mu^{n+1}$ where:

$$
\lambda_{j}^{n+1} \leftarrow \lambda_{j}^{n}+t^{n}\left(D_{j}-\sum_{i \in \mathcal{J}} Y_{i j}\right), \mu_{j s}^{n+1} \leftarrow \mu_{j s}^{n}+t^{n}\left(\sum_{i \in \mathcal{J}_{s}} Y_{i j}-\sum_{k \in \mathcal{K}} W_{j k s}-V_{j s}\right)
$$

The step size is determined by 


$$
t^{n}=\frac{\beta^{n}\left(B U B-\mathcal{L}^{n}\right)}{\sum_{j \in \mathcal{J}}\left(D_{j}-\sum_{i \in \mathcal{J}} Y_{i j}\right)^{2}+\sum_{s \in \mathcal{S}} \sum_{j \in \mathcal{J}}\left(\sum_{i \in \mathcal{J}_{S}} Y_{i j}-\sum_{k \in \mathcal{K}_{S}} W_{j k s}-V_{j s}\right)^{2}}
$$

Note that $\beta^{n}$ is a predetermined constant for the $n$th iteration, whose value will be halved if three consecutive iterations fail to make improvements.

The process of closing the gap between upper bound and lower bound is terminated if any of the following three criteria is satisfied:

- $\quad \frac{B U B-\mathcal{L}^{n}}{\mathcal{L}^{n}}<\varepsilon$ where $\varepsilon$ is a predetermined error tolerance

- $\quad \beta^{n}<\beta^{\text {min }}$, where $\beta^{\text {min }}$ is the minimal step size allowed

- $n>n^{\max }$, where $n^{\max }$ is the maximal step number allowed.

\subsection{Branch and Bound}

We incorporate the Lagrangian relaxation into a branch and bound algorithm to ensure the optimality gap is closed at $\varepsilon$-level. Since plants location decisions are more impactive than facility location decisions, we branch on the former (i.e. $X_{i}$ ) only. At each node, branching plant selected is the unfixed open location with greatest contribution $\phi(i)$. The variable $X_{j}$ is forced to be fixed value of zero and then one. Width-first search manner is applied in branching. A branch is fathomed if the lower bound of the parent node is greater than the best found upper bound. The tree is fathomed if obtained lower bound is within $\varepsilon$ times the best found upper bound, for which the latter is the $\varepsilon$-optimal solution for the original problem. In each node, final Lagrangian multipliers are inherited to its children nodes and used as initial multipliers.

\section{Computational Results}

In the first experiment, we compare total cost and computation time of three models. The first model locates both manufacturing plants and recall processing facilities, and allocates customer demands all in the first stage. In the second stage, the first model allocates returned products given possible recall scenarios in located plants. We use RNM (recovery network design model) as studied from Fleischmann et al. (2001) to represent the first model. The second model is our model $(\mathcal{F} \mathcal{L} \mathcal{R} \mathcal{P})$, which makes location-allocation decisions for plants and customers in the first stage, and locates facilities and allocates returned products in the second stage, considering the availability of reprocessing centers in various recall scenarios. The third model designs the most cost-effective network in a non-fail situation and takes the best available reprocessing centers when failure scenarios happen. We use reactive facility location model (RFL) to represent the third model setting. The first model RNM ignores the possibility that some facilities may not be available in some scenarios, and makes decisions of locating both plants and facilities in the first stage. On the contrary the neglected uncertainty is considered in $(\mathcal{F} \mathcal{L} \mathcal{R P})$, and we expect numerical results show the benefits of our consideration.

Our numerical example in Table 1 shows that the forward flow network of the first two 
models may not necessarily be the same. The second model shows better total cost (1\%-5\% less) and but a lot more complex to solve (using about five times more calculation time). Integration models (i.e. the first two models) performs better in total costs than reactive model (the third model). Location decisions are mostly different between integration models and reactive model.

Table 1. Compare costs and computation time of three models

\begin{tabular}{|l|l|l|l|l|l|l|l|l|l|}
\hline & RNM & $\mathcal{F} \mathcal{L} \mathcal{R} \mathcal{P}$ & RFL & RNM & $\mathcal{F} \mathcal{L} \mathcal{P} \mathcal{P}$ & RFL & RNM & $\mathcal{F} \mathcal{L} \mathcal{P} \mathcal{R}$ & RFL \\
\hline Cost $(\$ 000 \mathrm{~s})$ & 6.92 & 6.36 & 7.54 & 1.68 & 1.62 & 1.81 & 2.85 & 2.78 & 2.96 \\
\hline Time (sec.) & 0.61 & 0.24 & 0.50 & 0.92 & 0.98 & 0.70 & 6.64 & 24.58 & 2.56 \\
\hline Model size & $|\mathcal{J}|$ & 4 & & $|\mathcal{J}|$ & 8 & & $|\mathcal{J}|$ & 16 & \\
\hline & $|\mathcal{K}|$ & 3 & & $|\mathcal{K}|$ & 6 & & $|\mathcal{K}|$ & 12 & \\
\hline & $|\mathcal{S}|$ & 10 & & $|\mathcal{S}|$ & 36 & & $|\mathcal{S}|$ & 136 & \\
\hline & $|\mathcal{J}|$ & 4 & & $|\mathcal{J}|$ & 10 & & $|\mathcal{J}|$ & 20 & \\
\hline Cost $(/ \mathcal{F} \mathcal{L} \mathcal{R P})$ & 1.09 & 1.00 & 1.19 & 1.04 & 1.00 & 1.12 & 1.03 & 1.00 & 107 \\
\hline Time $(/ \mathcal{F} \mathcal{L} \mathcal{R P})$ & 2.56 & 1.00 & 2.11 & 0.94 & 1.00 & 0.71 & 0.27 & 1.00 & 0.10 \\
\hline
\end{tabular}

In this section we use numerical experiments to test the necessity and significance of product recall modeling as well as the impacts of parameters. The model is coded with General Algebraic Modeling System (GAMS) language and tested on a GAMS server which runs on Intel Xeon dual CPU $2.00 \mathrm{GHz} 2.00 \mathrm{GHz}$ processor with $8.0 \mathrm{~GB}$ RAM under Windows 64-bite operating system. We consider three variations in both environment settings and modeling settings in our experiments. Modeling settings describe the strategy of network design that a manager adopts, and environment settings simulate the true events for which the adopted strategic locations are used to satisfy demands and manage reverse flows. Depending on how many plants issue recalls in each second stage scenario, we consider three variations, namely no-recall, single-recall and dual-recall. No-recall does not consider the possibility of recall and its impact on the network. Single-recall assumes exactly one plant issues a recall in each scenario. Dual-recall considers the occurrence of recall and assumes at most two plants issues recalls per scenario, i.e., situations of exactly one plant and exactly two plants issuing recalls. Table 2 illustrates the relationship of no-recall, single-recall and dual-recall in environment and modeling settings.

Table 2. Relationship of no-recall, single-recall and dual-recall in environment and modeling settings

\begin{tabular}{|l|l|c|c|}
\hline & $\begin{array}{l}\text { Number of plants issue } \\
\text { recall per scenario }\end{array}$ & $\begin{array}{l}\text { Environment } \\
\text { notation }\end{array}$ & $\begin{array}{l}\text { Modeling } \\
\text { notation }\end{array}$ \\
\hline No-recall & 0 & $S 0$ & $M 0$ \\
\hline Single-recall & 1 & $S 1$ & $M 1$ \\
\hline Dual-recall & 1 OR 2 & $S 2$ & $M 2$ \\
\hline
\end{tabular}


Combination of three modeling settings and three environment settings gives us nine optimal total costs for a specific set of parameters. For instance, the expected total cost with (M0, S1) reflects the expenditure of both forward and reverse flows when there are recalls in reality, in fact one recall per scenario, while the manager assumes no recall in strategic network designs. Table 3 shows one set of typical experiment results, i.e., expected total costs of three model settings under three environments.

Table 3. Numerical example of optimal costs for three modeling settings under three environment settings

\begin{tabular}{|c|c|c|c|}
\hline & $S 0$ & $S 1$ & $S 2$ \\
\hline$M 0$ & $50,389.44$ & $142,210.15$ & $186,006.79$ \\
\hline M1 & $53,657.82$ & $138,413.01$ & $179,521.94$ \\
\hline M2 & $53,657.82$ & $138,413.01$ & $179,521.94$ \\
\hline
\end{tabular}

Notice diagonal numbers are the costs when model settings match environment settings, which we can call perfect information decisions (PIDs). Comparing with PIDs, total costs naturally increase when failing probability is either over-estimated (e.g., model considers at most two facilities have recalls per scenario while only one facility has recall in reality), or under-estimated (e.g., model considers no recall while in reality exactly one plant issues recall per scenario). We use regrets to evaluate effects of wrong estimation on total costs.

In the search of optimal modeling setting in three scenarios, we use Savage's minimax regrets method, a widely applied approach that conservatively chooses the option of least worst-case costs (Savage 1951). We define regrets in two ways: relative measure and absolute measure. Relative measure uses the percent cost increase when modeling settings mismatch environment settings compared to PIDs. Absolute measure uses value increase instead of percent increase. Table 4 shows results of applying relative and absolute measures of data in Table 3.

The reason that we adopt two measures to describe regrets is that PIDs under different environment settings can differ in significant ways. Therefore, the best modeling option reflected by relative measure could be different from the choice of absolute measure because the PID reference may change. Data in Table 4, for example, shows that, with relative measure, no-recall modeling (M0) is superior to single-recall (M1) and dual-recall modeling (M2) because, with M0, the worst cost increase is 3.61\% more than PIDs compared to $6.49 \%$ increase for both M1 and M2. However, with absolute measure, the opposite is true (i.e., with M0, the worst cost increase is $\$ 6485$ compared to $\$ 3268$ for both M1 and M2). To avoid bias generated by choosing only one measure, we use both measures. 
Table 4. Apply relative measure (left) and absolute measure (right)

\begin{tabular}{|l|l|l|l|l|l|l|}
\hline & \multicolumn{1}{|c|}{$S 0$} & \multicolumn{1}{c|}{$S 1$} & \multicolumn{1}{c|}{$S 2$} & \multicolumn{1}{c|}{$S 0$} & $S 1$ & $S 2$ \\
\hline$M 0$ & 1 & $102.74 \%$ & $103.61 \%$ & - & $3,797.14$ & $6,484.84$ \\
\hline$M 1$ & $106.49 \%$ & 1 & 1 & $3,268.38$ & - & \\
\hline$M 2$ & $106.49 \%$ & 1 & 1 & $3,268.38$ & & - \\
\hline
\end{tabular}

\subsection{Parameter Settings}

Our experiments test four sets of parameters settings: recall probability, capacity abundance, costly reverse flows and facility availability.

Based on the literature, the probability of a first stage plant issuing a recall in the second stage, recall probability, is within the range of $[0.01,0.08]$. Due to physical, social and financial differences, recall probabilities of candidate plant locations may be quite different from each other. We use a uniform distribution with the range [0.01, 0.08] to generate the probability of each plant incurring recalls.

Capacity abundance is how abundant one plant's capacity is compared to the total demands, i.e., the proportion of total demands that one plant can satisfy with its capacity. Four degrees of capacity abundance are considered as in Table 5.

Table 5. Capacity abundance settings

\begin{tabular}{|l|l|l|}
\hline Capacity abundance & Notation & Proportion of total demands that a facility can satisfy \\
\hline Tight & Cap1 & $25 \%$ \\
\hline Fair & Cap2 & $50 \%$ \\
\hline Medium & Cap3 & $75 \%$ \\
\hline Plenty & Cap4 & $100 \%$ \\
\hline
\end{tabular}

Costly reverse flows measure how expensive reverse flows are compare to forward flows. Three ratios are considered as in Table 6.

Table 6. Costly reverse flows settings

\begin{tabular}{|l|l|l|}
\hline Costly reverse flows & Notation & $\begin{array}{l}\text { Ratio of unit cost in reverse flows } \\
\text { compare to that of forward flows }\end{array}$ \\
\hline Inexpensive & costRF1 & 10 \\
\hline Medium & costRF2 & 50 \\
\hline Expensive & costRF3 & 100 \\
\hline
\end{tabular}

Facility availability measures the proportion of available facilities to open in the second stage compared to total number of possibly usable facilities. Four degrees of facility availability are considered as in Table 7. 
Table 7. Facility availability settings

\begin{tabular}{|l|l|l|}
\hline Facility availability & Notation & Percentage of facilities available to open in the second stage \\
\hline Scarce & prKs1 & $20 \%$ \\
\hline Somewhat & prKs2 & $40 \%$ \\
\hline Adequate & prKs3 & $60 \%$ \\
\hline Sufficient & prKs4 & $80 \%$ \\
\hline
\end{tabular}

\subsection{Results}

\subsubsection{Impacts of Facility Availability}

In order to find the impacts of facility availability on the choice of best modeling settings, we test four different levels of facility availability. With each level, we experiment with four variations of capacity abundance and three variations of costly reverse flows, applying randomly generated recall probabilities following a uniform distribution. Results are shown in Table 8.

Table 8. Proportion of optimal model setting under various facility availabilities

\begin{tabular}{|l|l|l|l|l|l|l|}
\hline & & prKs1 & prKs2 & prKs3 & prKs4 & Average \\
\hline Relative & $M 0$ & $12.20 \%$ & $10.70 \%$ & $11.52 \%$ & $12.15 \%$ & $11.64 \%$ \\
\hline measure & $M 1$ & $45.14 \%$ & $44.95 \%$ & $46.23 \%$ & $45.46 \%$ & $45.45 \%$ \\
\hline & $M 2$ & $42.66 \%$ & $44.35 \%$ & $42.25 \%$ & $42.39 \%$ & $42.91 \%$ \\
\hline Absolute & $M 0$ & $4.08 \%$ & $3.86 \%$ & $4.62 \%$ & $4.52 \%$ & $4.27 \%$ \\
\hline measure & $M 1$ & $48.62 \%$ & $49.20 \%$ & $49.86 \%$ & $49.46 \%$ & $49.29 \%$ \\
\hline & $M 2$ & $47.30 \%$ & $46.94 \%$ & $45.52 \%$ & $46.02 \%$ & $46.44 \%$ \\
\hline
\end{tabular}

When interpreting this table, note that the table columns for both relative and absolute measures sum to one. Each table entry represents the proportion of the time that the respective modeling setting is optimal. For example, using the relative measure, when facility availability is $20 \%$ (prKs1), the proportion of M1 being the optimal model is $45.14 \%$. We see that facility availability in the second stage does not significantly impact the choice of the best model setting using either relative or absolute measures (i.e., the values in each row do not deviate much from the row average). For example, in the relative measure M1 row, proportions range from $44.95 \%$ to $46.23 \%$ with an average of $45.45 \%$. Our prior is that decreasing facility availability could increase the proportion of modeling with recall considerations as optimal settings because higher facility availability indicates lower uncertainty in managing reverse flows and thus lower expected costs. Our results, on the contrary, show the optimal model setting is indifferent to facility availability. One explanation might be that our aggregation of data nullified the impact, or the impact is overshadowed by other more influential factors such as capacity abundance.

In all four cases, models considering recalls (M1UM2) performs significantly better than models without (M0) (e.g., for both relative and absolute measures, the M1 and M2 row 
values are much larger than the M0 ones). On average, with relative measure optimal models considering recalls, M1 and M2 account for $88.36 \%(45.45+42.91)$ of optimal model settings. With absolute measure, optimal models considering recalls account for $95.73 \%$ (49.29+46.44). This dominance proves that considering potential product recalls reduces total costs in the long run. Disregarding potential recalls could lead to selection of plant locations that initially seem to minimize costs, but that in hindsight are risky candidate sites with high expected costs to handle possible recalls. Our results with both relative and absolute measures support the assumption that designing with recall considerations minimizes the worst-case regrets.

The results also suggest that it is not obviously better to consider dual recall over single recall modeling. On average, of optimal models with recall consideration, using relative measure, single-recall models (M1) have proportions of $45.45 \%$ compared to $42.91 \%$ for dual-recall models; using absolute measure, single-recall models have $49.29 \%$ versus $46.44 \%$ for dual-recall models.

Dual recalls consider the following cases: a) two plants incurring recalls at the same, and b) only one plant incurring a recall at a time. Since plants incur recalls independently with a small probability, between $1 \%$ and $8 \%$, the chances of two recalls happening at the same time appears to be too small to impact the network design in any noticeable scale. However, dual recalls modeling requires much more computation resources compared to single recalls since the scenario size increases exponentially. Balancing the above considerations, single recall modeling sufficiently serves our purpose of planning for potential recall risks and rationalizing computation power.

\subsubsection{Impact of Capacity Abundance}

Risks of not considering recall in network design decrease when plants' capacity abundance increases. The reason may be that insufficient plants' capacity leads to various recall scenarios and thus increases the expected costs of managing recalls. This suggests abundant plants' capacity allows more space for risk control for managers in designing networks without recall concerns. Results concerning capacity abundance are shown in Table 9. When capacity is tight (Cap1), it is almost never good to ignore the possibility of recalls (i.e., for relative measure, the M0 table entry is $2 \%$ and for absolute measure, the M0 table entry is $0 \%$ ).

Table 9. Proportion of optimal model setting under various capacity availability

\begin{tabular}{|l|l|l|l|l|l|}
\hline & & Cap1 & Cap2 & Cap3 & Cap4 \\
\hline Relative & $M 0$ & $2.00 \%$ & $5.48 \%$ & $12.33 \%$ & $41.05 \%$ \\
\hline measure & $M 1$ & $50.22 \%$ & $47.95 \%$ & $44.57 \%$ & $32.31 \%$ \\
\hline & $M 2$ & $47.78 \%$ & $46.57 \%$ & $43.10 \%$ & $26.64 \%$ \\
\hline Absolute & $M 0$ & $0.00 \%$ & $5.00 \%$ & $0.48 \%$ & $20.71 \%$ \\
\hline measure & $M 1$ & $50.76 \%$ & $47.48 \%$ & $50.00 \%$ & $45.33 \%$ \\
\hline & $M 2$ & $49.24 \%$ & $47.52 \%$ & $49.52 \%$ & $33.95 \%$ \\
\hline
\end{tabular}




\section{I Macrothink}

Business and Economic Research

ISSN 2162-4860

2020, Vol. 10, No. 4

The only time that it might be acceptable for decision makers to ignore recall costs is when plant capacity is large compared to demand (i.e., when there is plenty of capacity (Cap4), the M0 table entry is $41.05 \%$ for relative measure and the M0 table entry is $20.71 \%$ for absolute measure). However, if the business is growing, current excess capacity will ultimately disappear. Therefore planning with potential recall serves the long term goal of building reliable and cost-effective networks.

\subsubsection{Impact of Costly Reverse Flows}

Results concerning capacity abundance are shown in Table 10. Dominance of M1andM2 may be attributable to the distinguishable recall probabilities of candidate plant locations. To optimize the network design for first stage only, $M 0$ may choose to open plants at relatively lower expense despite of their high chance of issuing recalls in the second stage, which results in premium payments when recalls do occur.

Table 10. Proportion of optimal model setting under various costly degrees of reverse flows

\begin{tabular}{|c|c|c|c|c|}
\hline & & $\mathrm{cRF} 1$ & $\mathrm{cRF} 2$ & $\mathrm{cRF} 3$ \\
\hline Relative & $M 0$ & $7.36 \%$ & $15.46 \%$ & $14.67 \%$ \\
\hline measure & $M 1$ & $49.17 \%$ & $43.26 \%$ & $42.81 \%$ \\
\hline & $M 2$ & $43.47 \%$ & $41.29 \%$ & $42.52 \%$ \\
\hline Absolute & $M 0$ & $2.86 \%$ & $4.58 \%$ & $5.57 \%$ \\
\hline measure & $M 1$ & $51.70 \%$ & $48.01 \%$ & $48.27 \%$ \\
\hline & $M 2$ & $45.44 \%$ & $47.41 \%$ & $46.16 \%$ \\
\hline
\end{tabular}

\subsubsection{Cost Increase as a Result of Neglecting Recalls in Network Design}

We apply the same set of recall probability and fix facility availability at $70 \%$. Numerical results show the dominance of $M 1$ andM2 over $M 0$ with minimax regrets similar to the results of Experiment 1. Results concerning capacity abundance are shown in Table 11.

Table 11. Max regrets for choosing M0 with relative measures

\begin{tabular}{|l|l|l|l|l|l|}
\hline Max regret & & Cap1 tight & Cap2 fair & Cap3 medium & Cap4 plentiful \\
\hline costRF1 & Inexpensive & $70 \%$ & $50 \%$ & $40 \%$ & $40 \%$ \\
\hline costRF2 & Medium & $90 \%$ & $70 \%$ & $60 \%$ & $60 \%$ \\
\hline costRF3 & Expensive & $100 \%$ & $80 \%$ & $70 \%$ & $70 \%$ \\
\hline
\end{tabular}

We also notice $M 1$ and $M 2$ have very close or equal total costs in various capacity abundance and costly reverse flow settings. Both perform dominantly better than $M 0$ when considering recalls.

We show the impact of capacity availability/costly reverse flow on the cost of $M 0$ compared to $M 1$ and $M 2$ with relative measure, which is the cost of neglecting recalls in network design. 
The costs of overlooking potential recalls vary largely from our randomly generated data sets, which indicates not only considering recalls in initial designs is necessary but also accurately predicting product recall probability can be crucial to effectively design the network.

\section{Conclusions}

This paper addresses the supply chain safety control issue by designing the supply chain network to incorporate the negative effects of product recalls. This work focuses on managing the reverse flow (recalled products) in a cost-efficient manner. We study the efficient way of managing random and rare major product recalls and consider how to quickly build a reprocessing network to dispose recalled products. We study the location-allocation problem with random occurrence of product recalls and treat the recall incidences as disruptions to the supply chain. Three features distinguish our problem from other location-allocation problems. Firstly, facility location and allocation decisions occur in two stages. Secondly, the second stage location-allocation happens under uncertainty. Thirdly, reverse logistic flows exist in the second stage.

We design a two-stage stochastic mixed integer programming model, in which we locate the manufacturing plants in the first stage and the reprocessing/disposal facilities in the second stage. We adopt a scenario-based approach to describe the uncertainty of major recall events that may happen in manufacturing plants as well as of availability of reprocessing facilities. Given the complexity induced by our nested facility location problem, we devise an algorithm based on Lagrangian relaxation to solve the uncapacitated case.

The existing literature treats reverse flow on day-to-day basis. This modeling approach does not serve major product recalls well. We fill the gap by designing an optimal network that can accommodate product returns in the context of major product recalls.

We compare total cost and computation time in the search of optimal modeling setting in three scenarios based on the minimax regrets method using both relative and absolute measures. Our experiments test four sets of parameters settings: recall probability, capacity abundance, costly reverse flows and facility availability. We find that facility availability in the second stage does not significantly impact the choice of the best model setting. However, we find that designing with recall considerations minimizes worst-case regrets. Moreover, considering potential product recalls reduces total costs in the long run - disregarding potential recalls could lead to selection of plant locations that initially seem to minimize costs, but that in hindsight are risky candidate sites with high expected costs to handle possible recalls. Risks of not considering recall in network design decrease when plants' capacity abundance increases.

\section{References}

Akkerman, R., Farahani, P., \& Grunow, M. (2010). Quality, safety and sustainability in food distribution: A review of quantitative operations management approaches and challenges. $O R$ Spectrum, 32(4), 863-904. https://doi.org/10.1007/s00291-010-0223-2

Charlebois, S., Massow, M. V., \& Pinto, W. (2015). Food recalls and risk perception: An 
exploratory case of the XL Foods and the biggest food recall in Canadian history. Journal of Food Products Marketing, 21(1), 27-43. https://doi.org/10.1080/10454446.2013.856055

Chvátal, V. (1983). Linear programming. Macmillan.

Dada, M., Petruzzi, N. C., \& Schwarz, L. B. (2007). A newsvendor's procurement problem when suppliers are unreliable. Manufacturing \& Service Operations Management, 9(1), 9-32. https://doi.org/10.1287/msom.1060.0128

Daskin, M. S. (2011). Network and discrete location: Models, algorithms, and applications. John Wiley \& Sons.

Desmarchelier, P., Fegan, N., Smale, N., \& Small, A. (2007). Managing safety and quality through the red meat chain. Meat Science, 77(1), 28-35.

https://doi.org/10.1016/j.meatsci.2007.04.027

Fisher, M. L. (1981). The lagrangian relaxation method for solving integer programming problems. Management Science, 27(1), 1-18. https://doi.org/10.1287/mnsc.27.1.1

Fleischmann, M., Beullens, P., Bloemhof-Ruwaard, J. M., \& Wassenhove, L. N. (2001). The impact of product recovery on logistics network design. Production and Operations Management, 10(2), 156-173. https://doi.org/10.1111/j.1937-5956.2001.tb00076.x

Lee, D. H., \& Dong, M. (2008). A heuristic approach to logistics network design for end-of-lease computer products recovery. Transportation Research Part E: Logistics and Transportation Review, 44(3), 455-474. https://doi.org/10.1016/j.tre.2006.11.003

Listeş, O. (2007). A generic stochastic model for supply-and-return network design. Computers and Operations Research, 34(2), 417-442.

https://doi.org/10.1016/j.cor.2005.03.007

Lu, Z., \& Bostel, N. (2007). A facility location model for logistics systems including reverse flows: The case of remanufacturing activities. Computers and Operations Research, 34(2), 299-323. https://doi.org/10.1016/j.cor.2005.03.002

Min, H., \& Ko, H. J. (2008). The dynamic design of a reverse logistics network from the perspective of third-party logistics service providers. International Journal of Production Economics, 113(1), 176-192. https://doi.org/10.1016/j.ijpe.2007.01.017

Pishvaee, M. S., Rabbani, M., \& Torabi, S. A. (2011). A robust optimization approach to closed-loop supply chain network design under uncertainty. Applied Mathematical Modelling, 35(2), 637-649. https://doi.org/10.1016/j.apm.2010.07.013

Qi, L. (2013). A continuous-review inventory model with random disruptions at the primary supplier. European Journal of Operational Research, 225(1), 59-74.

https://doi.org/10.1016/j.ejor.2012.09.035

Qi, L., Shen, Z. J. M., \& Snyder, L. V. (2010). The effect of supply disruptions on supply chain design decisions. Transportation Science, 44(2), 274-289.

https://doi.org/10.1287/trsc.1100.0320 


\section{Macrothink}

Business and Economic Research ISSN 2162-4860 2020, Vol. 10, No. 4

Ramezani, M., Bashiri, M., \& Tavakkoli-Moghaddam, R. (2013). A new multi-objective stochastic model for a forward/reverse logistic network design with responsiveness and quality level. Applied Mathematical Modelling, 37(1), 328-344.

https://doi.org/10.1016/j.apm.2012.02.032

Ruszczynski, A. P., \& Shapiro, A. (2003). Stochastic programming. Elsevier. https://doi.org/10.1016/S0927-0507(03)10001-1

Salema, M. I. A., Barbosa-Povoa, A. P., \& Novais, A. Q. (2006). A warehouse-based design model for reverse logistics. Journal of the Operational Research Society, 57(6), 615-629. https://doi.org/10.1057/palgrave.jors.2602035

Salema, M. I. G., Barbosa-Povoa, A. P., \& Novais, A. Q. (2007). An optimization model for the design of a capacitated multi-product reverse logistics network with uncertainty. European Journal of Operational Research, 179(3), 1063-1077.

https://doi.org/10.1016/j.ejor.2005.05.032

Savage, L. J. (1951). The theory of statistical decision. Journal of the American Statistical Association, 46(253), 55-67. https://doi.org/10.1080/01621459.1951.10500768

Savaskan, R. C., Bhattacharya, S., \& Van Wassenhove, L. N. (2004). Closed-Loop Supply Chain Models with Product Remanufacturing. Management Science, 50(2), 239-252.

https://doi.org/10.1287/mnsc.1030.0186

Snyder, L. V., \& Daskin, M. S. (2005). Reliability models for facility location: The expected failure cost case. Transportation Science, 39(3), 400-416.

https://doi.org/10.1287/trsc.1040.0107

Snyder, L. V., Scaparra, M. P., Daskin, M. S., \& Church, R. L. (2006). Planning for disruptions in supply chain networks. Tutorials in Operations Research, 2, 234-257.

\section{Copyright Disclaimer}

Copyright for this article is retained by the author(s), with first publication rights granted to the journal.

This is an open-access article distributed under the terms and conditions of the Creative Commons Attribution license (http://creativecommons.org/licenses/by/4.0/). 